\title{
Traveling waves in rotating Rayleigh-Bénard convection: Analysis of modes and mean flow
}

\author{
J. D. Scheel, * M. R. Paul, and M. C. Cross \\ Department of Physics, California Institute of Technology 114-36, Pasadena, California 91125, USA \\ P. F. Fischer \\ Mathematics and Computer Science Division, Argonne National Laboratory, Argonne, Illinois 60439, USA
}

(Received 14 May 2003; published 31 December 2003)

\begin{abstract}
Numerical simulations of the Boussinesq equations with rotation for realistic no-slip boundary conditions and a finite annular domain are presented. These simulations reproduce traveling waves observed experimentally. Traveling waves are studied near threshhold by using the complex Ginzburg-Landau equation (CGLE): a mode analysis enables the CGLE coefficients to be determined. The CGLE coefficients are compared with previous experimental and theoretical results. Mean flows are also computed and found to be more significant as the Prandtl number decreases (from $\sigma=6.4$ to $\sigma=1$ ). In addition, the mean flow around the outer radius of the annulus appears to be correlated with the mean flow around the inner radius.
\end{abstract}

DOI: $10.1103 /$ PhysRevE.68.066216

PACS number(s): 47.54.+r, 47.20.Bp, 47.32.-y, 47.27.Te

\section{INTRODUCTION}

One of the most productive systems for studying pattern formation is Rayleigh-Bénard convection [1]. In RayleighBénard convection, a fluid cell bounded by parallel plates is kept at a constant temperature difference $\Delta T$. This leads to a buoyancy-driven instability as $\Delta T$ increases past a critical value. In rotating convection, the entire cell is rotated about a vertical axis with a constant rotation rate $\Omega_{D}$. For certain choices of the parameters, traveling-wave wall modes have been found experimentally [2-5], and studied theoretically [6-11]. However, the nonlinear theories have assumed either free-slip boundary conditions and semi-infinite geometries $[6,9,10]$ or no-slip boundary conditions with periodic boundary conditions [7]. A recent theoretical analysis [11] has used realistic no-slip boundary conditions, but still neglects curvature effects. Here we use numerical simulations with noslip boundary conditions and finite annular geometries, with the same parameters that experimenters have used [4].

The system is modeled by the Boussinesq equations augmented by a Coriolis force [6]. The variables are nondimensionalized by specifying the length in terms of the cell height $d$, the temperature in terms of $\Delta T$, and the time in units of the vertical thermal diffusion time $\tau_{v}=d^{2} / \kappa$, where $\kappa$ is the thermal diffusivity. The equations are

$$
\begin{gathered}
\sigma^{-1}\left(\partial_{t}+\vec{u} \cdot \vec{\nabla}\right) \vec{u}=-\vec{\nabla} P+\nabla^{2} \vec{u}+R T \hat{z}+2 \Omega \vec{u} \times \hat{z}, \\
\left(\partial_{t}+\vec{u} \cdot \vec{\nabla}\right) T=\nabla^{2} T, \\
\vec{\nabla} \cdot \vec{u}=0 .
\end{gathered}
$$

The variable $\vec{u}(\vec{r}, t)$ is the velocity field, $P(\vec{r}, t)$ is the pressure, and $T(\vec{r}, t)$ is the temperature. The symbol $\partial_{t}$ indicates time differentiation and $\hat{z}$ is a unit vector in the vertical direction. The Prandtl number $\sigma=\nu / \kappa$, where $\nu$ is the kinematic viscosity. The Rayleigh number $R=\alpha g \Delta T d^{3} / \kappa \nu$,

*Electronic address: jscheel@ caltech.edu where $\alpha$ is the thermal-expansion coefficient and $g$ is the acceleration of gravity. The variable $\Omega$ is the dimensionless rotation rate $\left(\Omega=\Omega_{D} d^{2} / \nu\right)$. The aspect ratio $\Gamma$ is defined as the ratio of the radius of the cell to its depth $d$. The centrifugal force has been neglected because it is small relative to the gravitational force $\left(\Omega_{D}^{2} r / g \ll 1\right)$ for our rotation rates.

We have performed our numerical simulations of the Boussinesq equations using a parallel, spectral element code [12]. We used no-slip velocity boundary conditions along the walls (as written in a cylindrical coordinate system):

$$
u_{r}=u_{\phi}=u_{z}=0 \quad \text { at } \quad r=r_{\text {in }}, r_{\text {out }} \quad \text { and } \quad z=0,1 .
$$

For our temperature boundary conditions, we used insulating sidewalls and constant values along the top and bottom plates:

$$
\begin{gathered}
\partial_{r} T=0 \quad \text { at } r=r_{\text {in }}, r_{\text {out }}, \\
T=1 \quad \text { at } z=0, \quad T=0 \quad \text { at } z=1 .
\end{gathered}
$$

As the Rayleigh number is increased, for large enough rotation rates, the conduction profile gives way to a traveling-wave state localized along the walls. See Fig. 1 for examples. In the rotating frame, the inner and outer waves are counterpropagating, and the outer wave moves in the opposite direction of the rotation. As the Rayleigh number is increased even higher, this wall mode transitions to a bulk mode. In the case of zero rotation rate, patterns seen were similar to the results from simulations done by Sensoy and Greenside [13].

\section{COMPLEX GINZBURG-LANDAU EQUATION}

The wall mode is useful to study because, for large enough separation between inner and outer walls, the waves are mostly decoupled. Therefore, the outer wave (or inner) can be treated essentially as having only one spatial dimension. For all our cases, we analyzed the outer wave unless otherwise specified [14]. See Fig. 2 for examples of temperature versus angle data at fixed outer radius. 


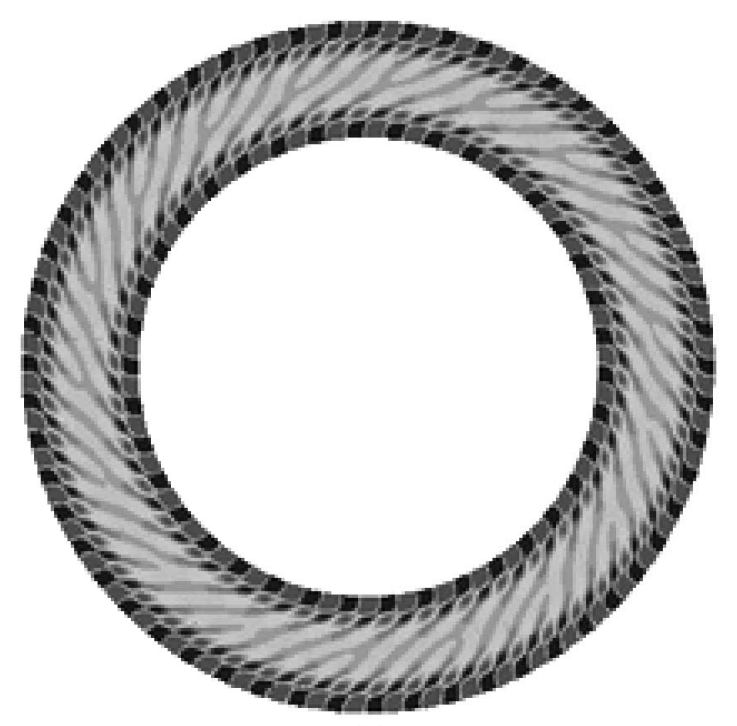

(a)

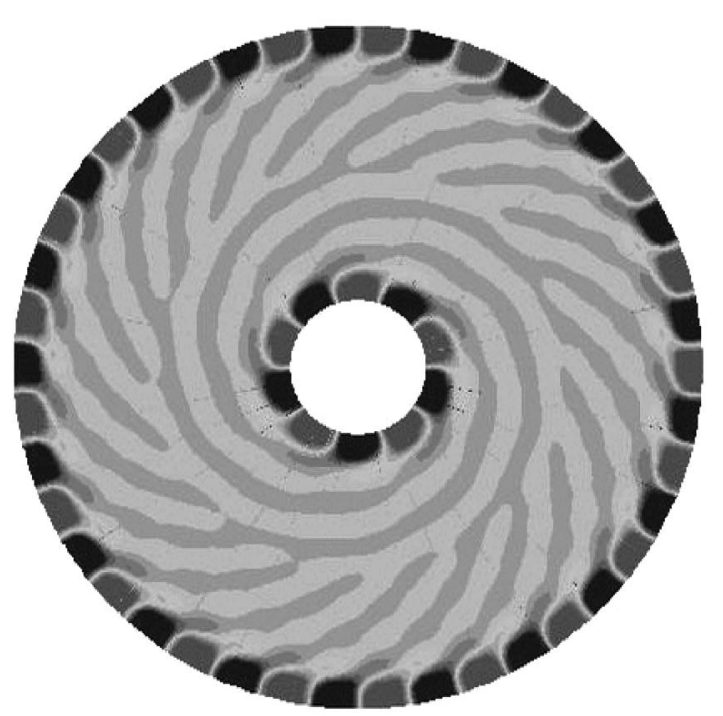

(b)

FIG. 1. Snapshot of temperature $T$ at the midplane, for traveling-wave wall modes with the following parameters: $\sigma=6.4$, and either (a) inner radius $=10$, outer radius $=15, \epsilon=0.017, \Omega=70$, $t=37$ or (b) inner radius $=1$, outer radius $=5, \epsilon=0.13, \Omega=274, t$ $=3$. The lightest gray in the center of the annular region denotes the conduction value $(T=0.5)$ and darker gray scale deviations give the values above and below this, which range from $T=0.49$ to 0.51 for (a), and 0.45 to 0.55 for (b). Note that the inner and outer waves are counterpropagating.

Near threshold, we can model this system with an amplitude equation [6]. We set the temperature deviation $\theta=T$ $-T_{o}$ [where $T_{o}(z)$ is the linear conduction profile] to be equal to

$$
\theta(\phi, r, z, t)=A(\phi, t) \exp \left(i\left[q_{c} r_{o} \phi-\omega_{c} t\right]\right) \chi(r, z)
$$

where $q_{c}$ is the critical wave number (i.e., the wave number at the onset of convection), $\omega_{c}$ is the critical precession fre- (a)

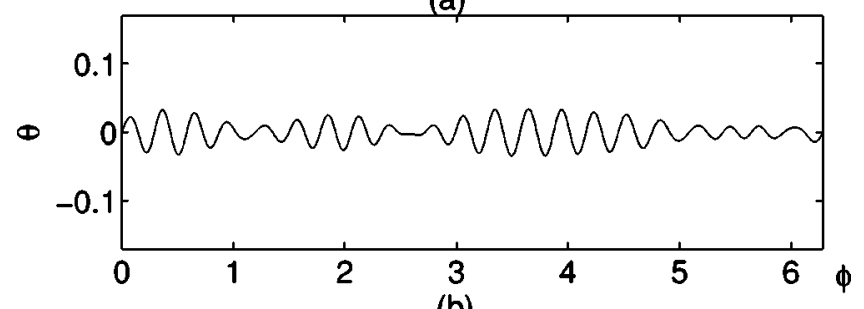

(b)

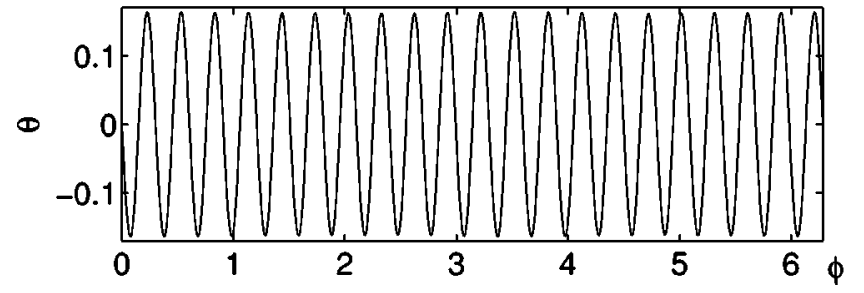

FIG. 2. Temperature deviations $\theta$ as a function of angle $\phi$ at fixed outer radius for (a) $t=0.8$, and (b) $t=3.44$. The following parameters were used: inner radius $=1$, outer radius $=5, \sigma=6.4$, $\epsilon=0.13, \Omega=274$.

quency, and $r_{o}$ is the outer radius. The function $\chi(r, z)$ represents the decay into the bulk, which is obtained from a linear stability analysis. Note that we have used the convention that the wave number $q=$ mode number $/ r_{o}$, since the decay length into the bulk is rather short, i.e., of the order of the depth. We then expect the complex amplitude $A$ to be described by the Complex Ginzburg-Landau equation (CGLE) [15-17]:

$$
\begin{aligned}
\tau_{o}\left(\partial_{t}+s \partial_{x}\right) A= & \epsilon\left(1+i c_{o}\right) A+\xi_{o}^{2}\left(1+i c_{1}\right) \partial_{x x} A \\
& -g\left(1+i c_{3}\right)|A|^{2} A,
\end{aligned}
$$

where $\partial_{x}$ represents partial differentiation with respect to $x$ $\equiv r_{o} \phi$. The small parameter $\epsilon=\left(R-R_{c}\right) / R_{c}$ represents the deviation from $R_{c}$, the critical Rayleigh number where conduction gives way to convection. The coefficients $\tau_{o}$ and $\xi_{o}$ are the characteristic time and length scales, respectively. The coefficient $s$ is the group velocity, $g$ gives the normalization of the amplitude, and $c_{o}$ is a constant that can be removed by transforming to a phase rotating frame. Since all the other coefficients can be scaled or transformed away, $c_{1}$ and $c_{3}$ essentially determine the behavior of the CGLE [18]. If $c_{1}=c_{3}$ or $c_{1}$ and $c_{3}$ are small, the equation is in the relaxational limit, where the CGLE reduces to the real Ginzburg-Landau equation and steady patterns are seen. If $c_{1} c_{3}+1<0$, the Newell criterion is reached, and solutions to the CGLE enter a spatiotemporal chaotic regime.

\section{Method of determining the coefficients}

The temperature deviations $\theta(\phi)$ for a fixed radius $r_{o}$, depth $z$, and time $t$ as shown in Fig. 2 can be spatially demodulated by Fourier analysis. By choosing random initial conditions, one can cause a rather large number of modes to be present. The amplitude of each individual mode as a function of time is shown in Fig. 3 for a representative case. At early times $(t \lesssim 0.75)$, one can see the growth (decay) of 


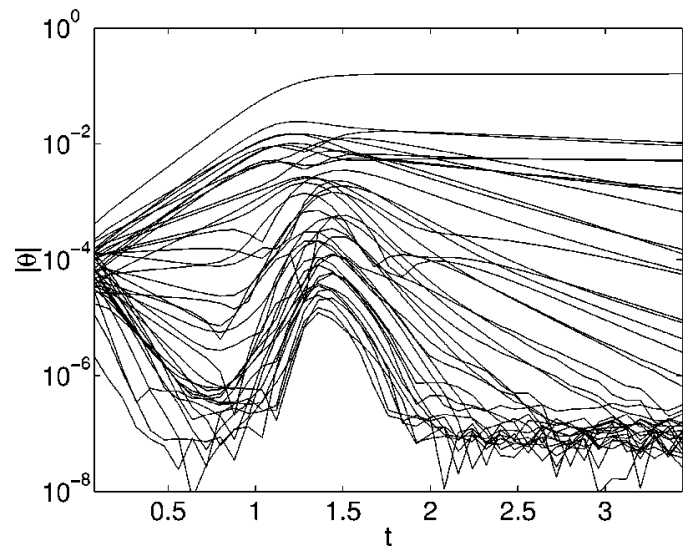

FIG. 3. Amplitudes $|\theta|$ of individual modes (obtained by Fourier analysis) vs time. Only the modes that have significant amplitudes are plotted, which range from $r_{o} q=2$ to $40\left(r_{o} q_{c}=21\right.$ here $)$. The parameters are as in Fig. 2. The largest amplitude mode corresponds to a mode number of 21 .

stable (unstable) modes. After some time has elapsed ( $t$ $\approx 1.5$ ), the nonlinearities cause this growth to saturate.

We can take advantage of these individual modes to determine the CGLE coefficients. In Fig. 4 we show the growth rates as a function of wave number for fixed $\epsilon$, which were determined by taking the slopes of each of the mode curves in the linear regime. Only modes with significant growth rates or small decay rates are shown in Fig. 4. By finding the roots of each of these curves, one can find the borders of the marginal stability diagram. This is shown in Fig. 5. For wave numbers inside the dashed line, the uniform state will be unstable to wall modes.

In Fig. 4, the curves merge together as $q \rightarrow 0$. This result is expected from the linearized, normal-mode analysis of the Boussinesq equations [19]. The growth rate approaches a constant value as $q$ approaches zero, independent of $\epsilon$.

Likewise the precession frequencies of each of the individual modes can be found from the rate of change of phase

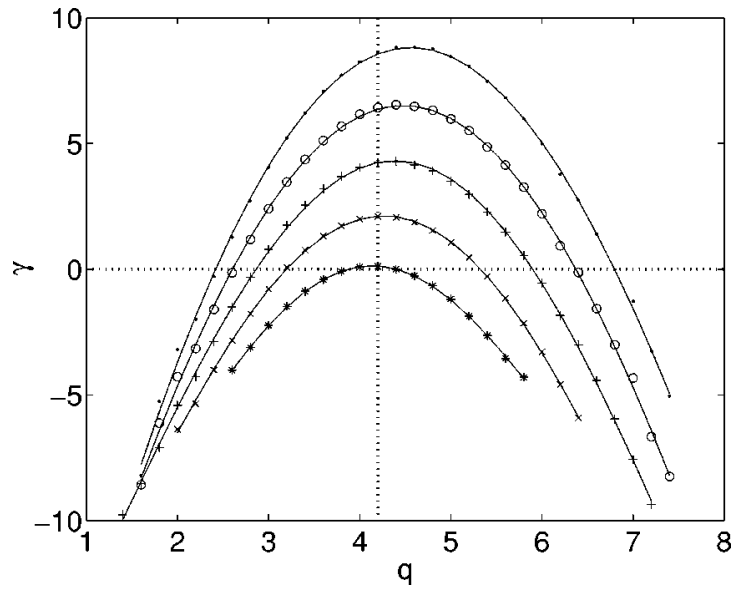

FIG. 4. Growth rates $\gamma$ as a function of wave number $q$ for the parameters as in Fig. 2. The markers correspond to the following values of $\epsilon$ : $* 0.003, x=0.05,+=0.10, o=0.15, \cdot=0.21$. The vertical dotted line is at $q=q_{c}=4.2$ (i.e., $r_{o} q_{c}=21$ ).

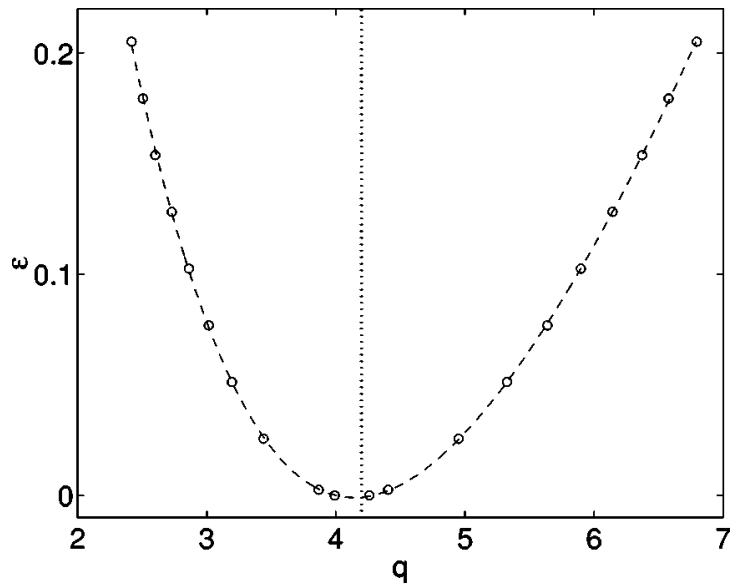

FIG. 5. Marginal stability diagram for the parameters as in Fig. 2. Data points were computed by finding the roots of the growth curves shown in Fig. 4. The dashed curve is a fourth-order polynomial fit. The vertical dotted line is at $q=q_{c}=4.2$.

with respect to time [the phase is also obtained from Fourier demodulation of $\theta(\phi)$ ]. Some representative cases are shown in Fig. 6. The negative sign on the precession frequency indicates that it is retrograde.

We now assume that the complex amplitude can be written as the sum of individual modes [20],

$$
A(\phi, t)=\sum_{k} a_{k} e^{\gamma_{k} t+i\left(k r_{o} \phi-\omega_{k} t\right)}
$$

The variable $a_{k}$ represents the amplitude of each mode, $\gamma_{k}$ is the growth rate, and $k$ and $\omega_{k}$ represent the difference in the wave number and precession frequency for that particular mode as referenced to the critical values $\left(q_{c}, \omega_{c}\right)$. If these are substituted into Eq. (8) and linearized, one obtains for each individual mode (by equating real and imaginary parts)

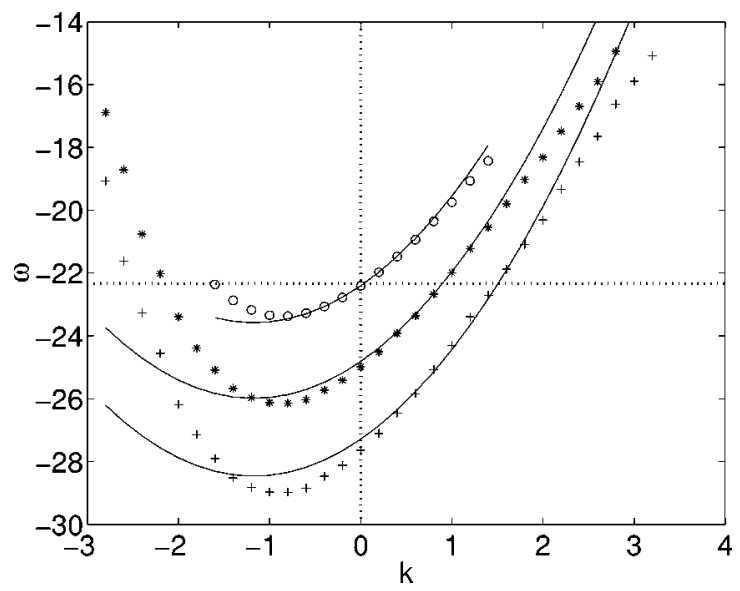

FIG. 6. Precession frequency $\omega$ as a function of reduced wave number $k=q-q_{c}$, where $q_{c}$ is the critical wave number (4.2 for this particular set of parameters-see Fig. 2). The markers correspond to $\epsilon$ 's: $\mathrm{o}=0.003, *=0.10,+=0.21$. The curves shown are the fits to Eq. (11), where the values of the CGLE coefficients are given in Table I. 


$$
\begin{gathered}
\gamma_{k}=\tau_{o}^{-1}\left(\epsilon-\xi_{o}^{2} k^{2}\right), \\
\omega_{k}=s k+\tau_{o}^{-1}\left(-\epsilon c_{o}+\xi_{o}^{2} c_{1} k^{2}\right) .
\end{gathered}
$$

If we drop the subscripts and instead think of $\gamma$ and $\omega$ as functions of $k$ as well as $\epsilon$, then we can compute all of the linear coefficients of the CGLE by taking various partial derivatives:

$$
\begin{gathered}
\frac{1}{\tau_{o}}=\left.\frac{\partial \gamma}{\partial \epsilon}\right|_{\epsilon=k=0}, \\
\xi_{o}^{2}=-\left.\frac{\tau_{o}}{2} \frac{\partial^{2} \gamma}{\partial k^{2}}\right|_{\epsilon=k=0}, \\
s=\left.\frac{\partial \omega}{\partial k}\right|_{\epsilon=k=0}, \\
c_{o}=-\left.\tau_{o} \frac{\partial \omega}{\partial \epsilon}\right|_{\epsilon=k=0}, \\
c_{1}=\left.\frac{\tau_{o}}{2 \xi_{o}^{2}} \frac{\partial^{2} \omega}{\partial k^{2}}\right|_{\epsilon=k=0} .
\end{gathered}
$$

Since we can calculate $\gamma(\epsilon, k)$ and $\omega(\epsilon, k)$ from (fourthorder) polynomial fits to the data (see Figs. 4 and 6), we can compute each of these coefficients [21].

The nonlinear coefficients $g, c_{3}$ can be found by using initial conditions so that only a single mode $k_{s}$ is excited. If the nonlinear terms are retained, the single mode versions of Eqs. (10) and (11) are

$$
\begin{gathered}
\gamma=\tau_{o}^{-1}\left(\epsilon-\xi_{o} k_{s}^{2}-g|a|^{2}\right), \\
\omega=s k_{s}+\tau_{o}^{-1}\left(-\epsilon c_{o}+\xi_{o} c_{1} k_{s}^{2}+g c_{3}|a|^{2}\right) .
\end{gathered}
$$

If we are far enough into the nonlinear regime, we can set the growth rate to zero and determine $g$,

$$
g=\left.\frac{\boldsymbol{\epsilon}}{\left|a^{2}\right|}\right|_{\gamma=k_{s}=0} .
$$

Likewise, if we eliminate $g$, we obtain

$$
c_{o}-c_{3}=-\left.\tau_{o} \frac{\partial \omega}{\partial \epsilon}\right|_{\gamma=\epsilon=k_{s}=0} .
$$

We use the normalization convention described in the Appendix. By combining Eqs. (19) and (A1), we can eliminate the amplitude and solve for $g$ by simply looking at how the Nusselt number $\mathcal{N}$ scales with $\epsilon$ :

$$
\mathcal{N}-1=\frac{c}{g} \epsilon
$$

The results are shown in Table I [22]. Agreement (except for the value of $g$ [23]) with the theory that uses no-slip
TABLE I. CGLE coefficients for the system parameters given in Fig. 4. Comparisons are made with experiment $(\mathrm{LE}=\mathrm{Liu}$ and Ecke [4]) and theory $(\mathrm{KC}=\mathrm{Kuo}$ and Cross [6], P=Plaut [Table I of Ref. [11]]. Also note we found $c_{0}=0.60, c_{1}=0.44, c_{3}=0.12$.

\begin{tabular}{lcccc}
\hline \hline & $\begin{array}{c}\text { Present } \\
\text { work }\end{array}$ & LE & KC & P \\
\hline$R_{c}$ & 19500 & 20850 & 19500 & 19660 \\
$q_{c}$ & 4.2 & 4.65 & 4.00 & 4.22 \\
$\omega_{c}$ & -22.3 & -22.0 & -24.0 & -22.4 \\
$\tau_{o}$ & 0.025 & .03 & 0.026 & 0.025 \\
$\xi_{o}$ & 0.22 & 0.179 & 0.24 & 0.21 \\
$s$ & 2.0 & 2.65 & 2.22 & 1.91 \\
$g$ & 1.63 & 0.74 & 1.11 & 0.53 \\
$\tau_{o}^{-1}\left(c_{o}-c_{1}\right)$ & 6.4 & 4.2 & 14.4 & 6.40 \\
$\tau_{o}^{-1}\left(c_{o}-c_{3}\right)$ & 19.3 & 20.4 & 19.2 & 19.7 \\
\hline \hline
\end{tabular}

velocity boundary conditions [11] is very good, whereas there is understandably some disagreement with the theory that employed free-slip velocity boundary conditions [6]. We find only partial agreement with the experimental results. However, the coefficients are all extremely sensitive to the fit, as can been seen in Fig. 6. Representative data for precession frequency are plotted along with a fit to the linearized CGLE (11) with the values of the coefficients given in Table I. The fits are good only very close to threshold (small $\epsilon$ and small $k$ ). Higher-order corrections to the CGLE would improve the fits, as was appropriately explored in Ref. [4].

\section{MEAN FLOW}

Here we explore the role mean flow plays in rotating convection. Theoretically one expects mean flow to be important for multiply connected domains like an annulus [11,25]. Mean flow in nonrotating convection arises from large-scale variations in local wave number and amplitude, which cause nonlocal pressure gradients [26,27]. For rotating convection, the traveling wave propagation will also drive a mean flow [28].

We calculated the total mean flow for our system by performing an average of the horizontal velocity $\vec{u}_{\perp}=\left(u_{r}, u_{\phi}\right)$ over the depth and the radius:

$$
\langle\vec{u}(\phi, t)\rangle_{r, z}=\int_{r_{\text {in }}}^{r_{\text {out }}} d r \int_{0}^{1} d z \vec{u}_{\perp}(r, \phi, z, t) .
$$

Often it is useful to separate the mean flow for the inner and outer waves, by performing the radial average only to the radius half way between the inner and outer radii. Because the traveling waves decay exponentially into the bulk, these half-averaged mean flows are a good measure of the inner and outer mean flows. For our traveling-wave trials (when the inner and outer waves are decoupled), the mean flow is observed to be in the same direction as the phase velocity of the waves, so the outer mean flow is retrograde and the inner flow is in the same direction as the rotation.

Results for various geometries and parameters are shown in Table II. Mean flow is present in all cases, but it becomes 
TABLE II. Tangential mean flow results. The fluctuating components in the $\phi$ direction (see Fig. 7) have been averaged over for inner and outer mean flows. The velocities are scaled by $\alpha$ as defined in Eq. (A3). The root-mean-square convection velocity $u_{\text {rms }}$ is given in the last column. In all cases, traveling waves were stable, and random initial conditions were used.

\begin{tabular}{lcccccccc}
\hline \hline$r_{\text {in }}, r_{\text {out }}$ & $R$ & $\Omega$ & $\sigma$ & $t$ & $\begin{array}{c}\alpha\left\langle u_{\phi}\right\rangle_{z, r, \phi} \\
\text { Inner half }\end{array}$ & $\begin{array}{c}\alpha\left\langle u_{\phi}\right\rangle_{z, r, \phi} \\
\text { Outer half }\end{array}$ & $\begin{array}{c}\alpha\left\langle u_{\phi}\right\rangle_{z, r} \\
\text { Total }\end{array}$ & $u_{\text {rms }}$ \\
\hline 1,5 & 23500 & 274 & 6.40 & 3.0 & 0.334 & -0.448 & -0.114 & 2.774 \\
1,5 & 23500 & 274 & 1.00 & 3.0 & 0.650 & -0.918 & -0.268 & 3.194 \\
10,15 & 23500 & 274 & 6.40 & 3.0 & 0.423 & -0.438 & -0.015 & 2.702 \\
10,15 & 23500 & 274 & 1.00 & 3.0 & 0.820 & -0.875 & -0.055 & 3.026 \\
10,15 & 6500 & 70 & 6.40 & 12.0 & 0.205 & -0.220 & -0.015 & 2.340 \\
\hline \hline
\end{tabular}

more significant for smaller Prandtl number. Also, the $\phi$ component of total mean flow (22) is nonzero but decreases as the aspect ratio increases. This result indicates that the total tangential mean flow is due to the fractional difference in radii between the inner and outer waves, and the total tangential mean flow should become zero as the aspect ratio goes to infinity. The last line of Table II corresponds to an $\epsilon$ of 0.08 , and is included for comparison with Plaut's theoretical calculations shown in Fig. 5 of Ref. [11]. The parameters used in Ref. [11] are $\Omega=100, \sigma=6.3, \epsilon=0.1, r_{\text {out }}-r_{\text {in }}=3$. If we radially average the azimuthal component of the largescale mean flow data over the outer half of the geometrical domain used in Ref. [11], we obtain a value of $\approx-0.4$. This agrees to within $5 \%$ of our value for the outer half mean flow if we take into account the scaling discrepancy in $g$ from Table I and hence $\alpha$ in Eq. (A3).

The outer and inner wave tangential mean flows have small fluctuating components in the angular coordinate as seen in Fig. 7. These components are equal and opposite, as they must be, to satisfy incompressibility. Thus, the tangential component of the total mean flow $\left\langle u_{\phi}\right\rangle_{r, z}$ is independent of the angular coordinate $\phi$. The presence of these fluctuations indicates that the outer and inner mean flows are correlated. The fluctuations are not seen when only a single

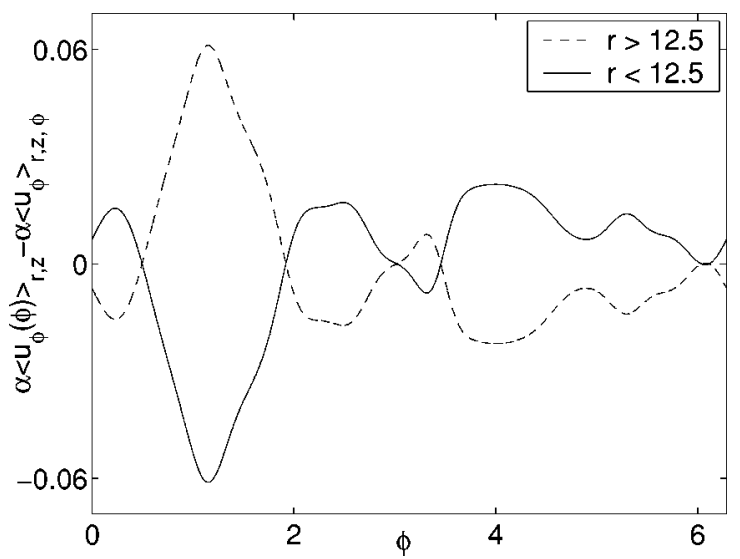

FIG. 7. Tangential mean flow averaged over the outer (or inner) half of the annulus, where the average over the angular coordinate $\phi$ has been subtracted for ease of comparison (see Table II for these averages). Data are for inner radius $=10$, outer radius $=15, \sigma$ $=1.0, R=23500, \Omega=274, t=3$. mode is present (obtained by starting with initial conditions resembling the desired mode). Defects and large-scale wave number variations are thought to be a reason for the mean flow correlations. This aspect of mean flow is being investigated.

\section{CONCLUSION}

Traveling-wave wall states were observed in numerical simulations of the Boussinesq equations with rotation for experimentally realistic geometries and boundaries. The CGLE coefficients were computed and agree (except for the value of $g$ ) with experiment and theory. So far the coefficients found have been close to the relaxational limit $\left(c_{1}, c_{3}\right.$ small), where coherent patterns are expected. A search is currently being conducted in the parameter range where van Hecke and van Saarloos suggest chaotic dynamics exist [9], that is where $c_{1} c_{3}<-1$.

Mean flow was also calculated and seen to be significant for a rotating annulus. Mean flow correlations were observed, which provide a long-range coupling between inner and outer waves that otherwise would be exponentially small. This phenomenon will be investigated, in addition to looking at nonuniform thermal boundary conditions that may lead to interacting waves. We also plan to use a technique of quenching the mean flow $[29,30]$ to see what effect it has on the traveling-wave patterns, and in particular whether it causes or suppresses any chaotic motion.

\section{ACKNOWLEDGMENTS}

We wish to thank M. van Hecke, R. E. Ecke, and K.-H. Chiam for insightful comments. This work was supported by the Engineering Research Program of the Office of Basic Energy Sciences at the Department of Energy, Grants Nos. DE-FG03-98ER14891 and DE-FG02-98ER14892, and the Mathematical, Information and Computational Sciences Division subprogram of the Office of Advanced Scientific Computing Research, U.S. Department of Energy, under Contract No. W-31-109-Eng-38. We also acknowledge the North Carolina Supercomputing Center and the Caltech Center for Advanced Computing Research. 


\section{APPENDIX: SCALING}

The magnitude of the amplitude $|A|$ depends on the choice of normalization for the linear mode $\chi(r, z)$. Typically the scaling of the amplitude is defined so that the Nusselt number $\mathcal{N}$ is proportional to the amplitude squared $|A|^{2}$,

$$
\mathcal{N}-1=|A|^{2} c .
$$

The Nusselt number is the ratio of the total heat flux to the heat flux in the conduction regime. For the nonrotating case, since the convection extends over the whole cell, $c$ is chosen to be unity. In the case of traveling waves, the convention has been to use a scale factor $c$ corresponding to the ratio of the area occupied by the waves to the total area of the cell [4], so we define our proportionality constant $c$ to be

$$
c=\left(\frac{\left(2 r_{\mathrm{out}}-1\right)+\left(2 r_{\mathrm{in}}+1\right)}{r_{\mathrm{out}}^{2}-r_{\mathrm{in}}^{2}}\right),
$$

where we have estimated the penetration of the wave in the radial direction to be 1 (in depth units). As a result, we can define a scaling constant

$$
\alpha=\sqrt{\left.\frac{\mathcal{N}-1}{c|A|^{2}}\right|_{t \rightarrow \infty}} .
$$

[1] M.C. Cross and P.C. Hohenberg, Rev. Mod. Phys. 65, 851 (1993).

[2] F. Zhong and R.E. Ecke, Chaos 2, 163 (1992).

[3] L. Ning and R.E. Ecke, Phys. Rev. E 47, 3326 (1993).

[4] Y. Liu and R.E. Ecke, Phys. Rev. E 59, 4091 (1999).

[5] K.M.S. Bajaj, G. Ahlers, and W. Pesch, Phys. Rev. E 65, 056309 (2002).

[6] E.Y. Kuo and M.C. Cross, Phys. Rev. E 47, R2245 (1993).

[7] T. Clune and E. Knobloch, Phys. Rev. E 47, 2536 (1992).

[8] H.F. Goldstein, E. Knobloch, I. Mercader, and M. Net, J. Fluid Mech. 248, 583 (1993).

[9] M. van Hecke and W. van Saarloos, Phys. Rev. E 55, R1259 (1997).

[10] J. Herrmann and F.H. Busse, J. Fluid Mech. 255, 183 (1993).

[11] E. Plaut, Phys. Rev. E 67, 046303 (2003).

[12] P.F. Fischer, J. Comput. Phys. 133, 84 (1997).

[13] B. Sensoy and H. Greenside, Phys. Rev. E 64, 046204 (2001).

[14] Since the traveling waves have a decay length of the order of the depth of the fluid $d$, the inner and outer waves are decoupled for the annular geometries used. Runs were made with conducting temperature boundary conditions on the inner wall instead of insulating (which suppresses wall modes), and outer wave results were unchanged.

[15] W. van Saarloos, in Spatio-Temporal Patterns, edited by P.E. Claudius and P. Palffy-Muhoray (Addison-Wesley, Reading, MA, 1995), pp. 19-31.

[16] W. van Saarloos and P.C. Hohenberg, Physica D 56, 303 (1992).

[17] I.S. Aranson and L. Kramer, Rev. Mod. Phys. 74, 99 (2002).
[18] M. van Hecke, Ph.D. thesis, Universiteit Leiden, 1996 (unpublished).

[19] P. Manneville, Dissipative Structures and Weak Turbulence (Academic Press, New York, 1990).

[20] E.Y. Kuo, Ph.D. thesis, California Institute of Technology 1994.

[21] The characteristic length scale $\xi_{o}$ can also be found from the marginal stability diagram, by noting that the boundaries of the stability diagram were found from setting the growth rate in Eq. (10) to zero: $\xi_{o}^{2}=\frac{1}{2} \partial^{2} \epsilon /\left.\partial k^{2}\right|_{\gamma=k=0}$.

[22] Since we have a finite system and have defined $q r_{o}=$ mode number, a change in $r_{o}$ (say of order $1 / 2$ the penetration depth) would cause a $10 \%$ change in $q_{c}, \xi_{o}$, and $s$. All other coefficients would be unaffected.

[23] We cannot explain this discrepancy. Our reduced Nusselt numbers for a given $\epsilon$ are about half as big as the data quoted by Liu and Ecke [4] on page 4093. Our Nusselt number versus $\epsilon$ calculations do agree to within $4 \%$ of Behringer and Ahler's results [24] for a nonrotating cell of aspect ratio 4.72 near threshold.

[24] R.P. Behringer and G. Ahlers, J. Fluid Mech. 125, 219 (1982).

[25] E. Plaut and F.H. Busse, J. Fluid Mech. 464, 345 (2002).

[26] A.C. Newell, T. Passot, and M. Souli, J. Fluid Mech. 220, 187 (1990).

[27] M.C. Cross and A.C. Newell, Physica D 10, 299 (1984).

[28] J.T. Stuart, J. Fluid Mech. 4, 1 (1958).

[29] M.R. Paul, M.C. Cross, and P.F. Fischer, Phys. Rev. E 66, 046210 (2002).

[30] K.-H. Chiam, M.R. Paul, M.C. Cross, and H.S. Greenside Phys. Rev. E 67, 056206 (2003). 